\title{
Syphilitic lymphadenitis clinically and histologically mimicking lymphogranuloma venereum
}

\begin{abstract}
A Wessels, C Bamford, D A Lewis, M Martini, H C Wainwright
Dr Annesu Wessels is a senior registrar in anatomical pathology working at Groote Schuur Hospital, Cape Town, South Africa. Dr Colleen Bamford is a senior pathologist in the Division of Microbiology, Faculty of Health Sciences, University of Cape Town. She is actively involved in research and education regarding the rational use of antibiotics and appropriate use of microbiological investigations. Prof. David Lewis is a sexual health physician currently working as Director of the Western Sydney Sexual Health Centre, Australia, with conjoint professorial appointment at the University of Sydney. He also serves as President of the International Union against Sexually Transmitted Infections, and was formerly Head of the Centre for HIV and Sexually Transmitted Infections at the National Institute for Communicable Diseases in South Africa. He has an interest in sexual health and has published more than 100 scientific articles. Dr Markus Martini is a medical officer in the Department of Surgery at Victoria Hospital, Wynberg, Cape Town. He has a special interest in laparoscopic surgery. Prof. Helen Wainwright is a principal pathologist and associate professor in the Department of Anatomical Pathology at the University of Cape Town. She has special interests in neonatal, hepatic and pulmonary pathology.
\end{abstract}

Corresponding author: A Wessels (wssann003@myuct.ac.za)

An inguinal lymph node was discovered incidentally during surgery for a suspected strangulated inguinal hernia. The patient had recently been treated for candidal balanoposthitis and was known to have a paraphimosis. A new foreskin ulcer was discovered when he was admitted for the hernia surgery. The lymph node histology showed stellate abscesses suggestive of lymphogranuloma venereum (LGV). Chlamydial serologic tests were negative. As the histological appearance and clinical details provided were thought to suggest LGV, tissue was also sent for a real-time quadriplex polymerase chain reaction assay. This was used to screen for Chlamydia trachomatis in conjunction with other genital ulcer-related pathogens. The assay was negative for C. trachomatis, but positive for Treponema pallidum. Further histochemical staining of the histological specimen confirmed the presence of spirochaetes.

S Afr Med J 2016;106(5):465-467. DOI:10.7196/SAMJ.2016v106i5.8723

A 56-year-old non-insulin dependent diabetic man initially presented with paraphimosis and candidal balanoposthitis. The treatment was successful and circumcision was planned, but the patient failed to return for surgery. Seven months later he presented acutely with a 2-day history of severe abdominal pain, nausea and constipation, and a 6-week history of increasing swelling in his right groin. These clinical features were indicative of a strangulated inguinal or femoral hernia. A small ulcer on his foreskin was also noted, while the phimosis was still present. A small uncomplicated direct inguinal hernia and a firm enlarged inguinal lymph node were found in surgery. The lymph node was excised for histology and microbiological culture, while a routine open mesh repair of the hernia and a circumcision were performed. The foreskin was not submitted for histology. The patient recovered well after surgery.

On gross examination, the lymph node measured $35 \times 25 \times$ $20 \mathrm{~mm}$, and showed well-circumscribed pale areas on the cut section. The tissue was fixed and embedded in paraffin. Sections were stained routinely with haematoxylin and eosin, and examined using light microscopy.

Microscopically, the sections showed a diffusely enlarged lymph node and adjacent fibroadipose tissue. The nodal architecture was effaced by necrotising inflammation producing stellate and serpiginous abscesses containing macrophages, neutrophils and nuclear debris without giant cells or granulomas (Fig. 1). No organisms were demonstrated on histochemical stains (Ziehl-Neelsen \pm modification, PAS, Grocott).

Bacterial and mycobacterial cultures of the lymph node tissue yielded no growth. Chlamydial serology was requested and a complement fixation test (CFT) showed low-level positive antibody titres of 1 in 32 for the chlamydia-specific panel, which includes

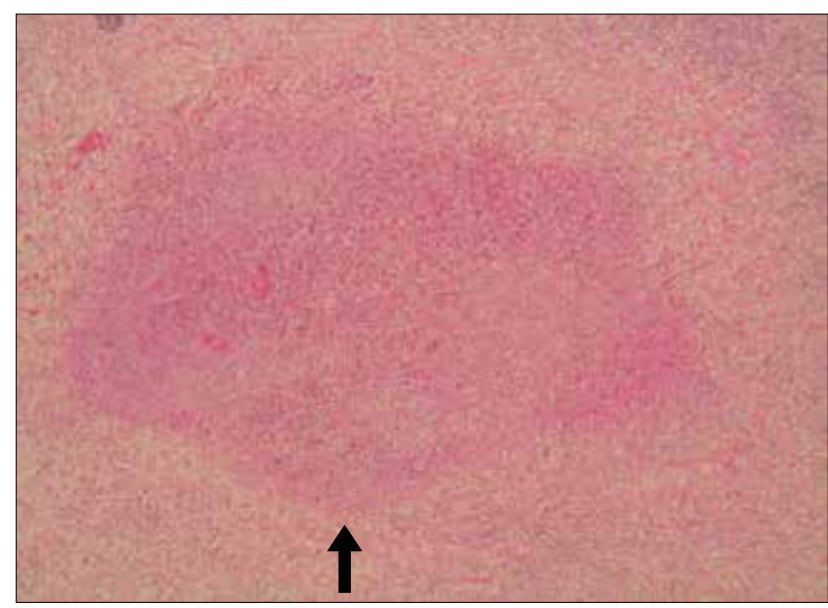

Fig. 1. Haematoxylin and eosin-stained section of the lymph node showing a stellate-shaped abscess (arrow).

Chlamydia trachomatis (serovars A-K and L1-L3), Chlamydophila psittaci and Chlamydophila pneumoniae. This low-level positivity can be seen as a result of previous exposure to chlamydial infection, and cross-reactivity between antigens is frequently encountered. A subsequent $C$. trachomatis microimmunofluorescence test was negative for antibodies against lymphogranuloma venereum (LGV)specific serovars, essentially ruling out LGV as a diagnosis.

As the histological appearance and the clinical details provided were thought to be suspicious for LGV, residual inguinal lymph node tissue was sent to the Centre for HIV and Sexually Transmitted Infections at the National Institute for Communicable Diseases 
in Johannesburg, South Africa (SA) for confirmation and further testing. A validated in-house, real-time quadriplex polymerase chain reaction (PCR) assay was used to screen for C. trachomatis in conjunction with other genital ulcer-related pathogens, specifically herpes simplex virus (HSV), Treponema pallidum and Haemophilus ducreyi. The assay was negative for C. trachomatis, HSV and H. ducreyi, but positive for T. pallidum. We were unable to test the genital ulcer for the presence of T. pallidum with the same molecular assay as the foreskin had been discarded post circumcision.

Given the positive PCR assay result, a Warthin-Starry stain was requested on the histologically processed tissue biopsy and found to be positive for spirochaetes (Fig. 2). In addition, rapid plasma reagin (RPR) and a T. pallidum haemagglutination assay were performed retrospectively. Both were reactive, with titres of 1:16 and 1:1 280, respectively. A combination of molecular, histological and serological diagnostic strategies confirmed a clinically unexpected diagnosis of syphilis in the patient.

\section{Discussion}

The presence of a mass in the groin area, especially of short duration, would prompt the clinician to investigate the patient for an incarcerated inguinal hernia, among other things. Finding enlarged lymph nodes and adjacent fibroadipose tissue in such cases is not uncommon. Pathological changes in the aforementioned lymph nodes are also not unusual as this is frequently the reason for the incarceration.

Necrotising lymphadenitis can be a nonspecific finding accompanying many benign and malignant processes involving lymph nodes at any site. Acute nonspecific lymphadenitis, which is rarely biopsied, shows dilation of sinuses, neutrophilic infiltrates, vascular ectasia and oedema. Suppurative lymphadenitis may be due to a Staphylococcus aureus infection, mesenteric lymphadenitis, LGV and cat-scratch disease. Necrotising lymphadenitis may be seen in bubonic plague, tularaemia, anthrax, typhoid fever, melioidosis and Kikuchi's lymphadenitis. ${ }^{[1]}$

The presence of stellate micro-abscesses is a more specific finding, with a differential diagnosis of cat-scratch disease, LGV, and less likely tularaemia and atypical mycobacterial infection. ${ }^{[2]}$ The histological findings in this case represent a very unusual lymph node response to syphilis. Typical features of syphilis, such as endarteritis and phlebitis, were not easy to see in this particular case due to extensive necrosis.

The famous physician Sir William Osler is quoted as saying 'he who knows syphilis knows medicine. ${ }^{[3]}$ Our case is another good example of the clinical and pathological mimicry that characterises infection with T. pallidum. Before the introduction of penicillin, which led to a marked reduction in syphilitic infections, every clinician was well attuned to the myriad clinical presentations of this important infectious disease. These days, however, syphilis may be misdiagnosed and mistreated if the condition itself is not considered in the differential diagnosis. ${ }^{[3]}$ Key to making a correct diagnosis is the taking of an adequate sexual history which tends to be poorly undertaken in many medical and surgical specialities. The risk of syphilis is increased in patients with multiple sexual partners, among men who have sex with sex workers, among sex workers themselves, and importantly among those men who have sex with men (MSM) who are engaged in high-risk sexual practices. ${ }^{[4-6]}$ Syphilis may be easily transmitted through vaginal, anal and, of note, oral sexual intercourse. $^{[3]}$

Untreated syphilis evolves through several stages. Localised lymph node enlargement can be seen in primary or tertiary syphilis, whereas secondary syphilis commonly shows generalised lymphadenopathy in keeping with the generalised dissemination of treponemes that accompanies this stage of the disease.

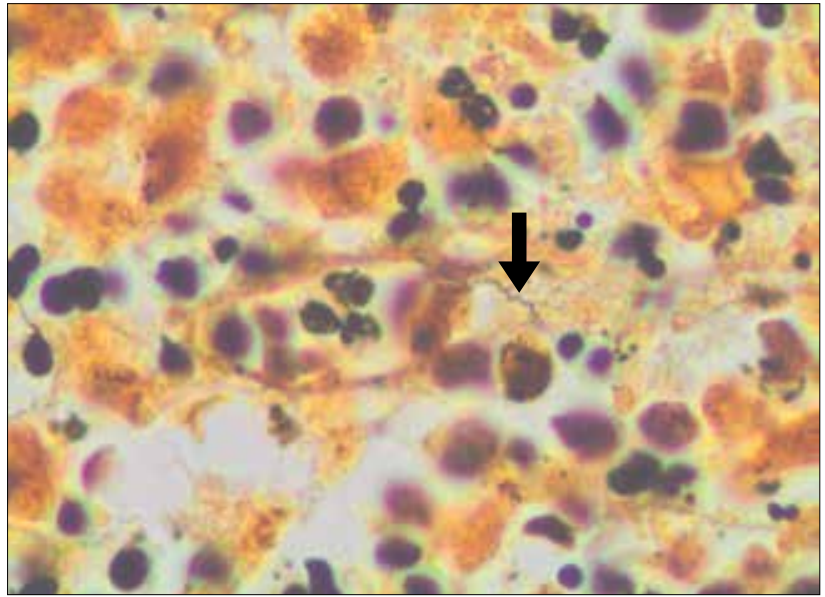

Fig. 2. Warthin-Starry histochemical stain showing a coiled spirochaete (arrow) morphologically consistent with $\mathrm{T}$. pallidum.

The primary ulcers may be painless and may therefore go unnoticed by the patient. ${ }^{[5]}$ The associated inflammation that accompanies many primary lesions may cause tissue swelling and occasionally phimosis, which interferes with clear visualisation of the ulcer. Histologically, primary nodal syphilis usually presents with capsular and pericapsular inflammation and extensive fibrosis. A diffuse plasmacytic infiltrate, proliferation of blood vessels with endothelial swelling and phlebitis/endarteritis are commonly seen, in a background of follicular hyperplasia. ${ }^{[1,2]}$ Rarely, non-caseating granulomas and abscesses can be found. ${ }^{[1]}$ As with this case, T. pallidum can be demonstrated by histochemical stains (WarthinStarry, Levaditi), immunohistochemistry or immunofluorescence. ${ }^{[2]}$ Detection in fine-needle biopsies of lymph nodes using Southern blotting and PCR is also possible..$^{[2]}$

If available, molecular assays are the diagnostic test of choice for primary syphilis. ${ }^{[5]}$ It is important to note that sera from up to a third of patients with primary syphilis will be non-reactive when tested with non-treponemal (such as RPR) and treponemalspecific serological assays. Therefore, negative syphilis serology in the presence of a genital ulcer does not exclude syphilis as a diagnosis and blood should be retested after a further 10 - 14 days. In the secondary stage, all serological tests should be positive for syphilis and the diagnosis is therefore easy to confirm as long as the clinician considers the diagnosis.

LGV is a less common sexually transmitted disease and is caused by the lymphotropic serovars of $C$. trachomatis (L1, L2, L3 and their variants). ${ }^{[7]} \mathrm{A}$ small, painless genital vesicle or ulcer may be the presenting sign (primary stage) although this is often missed. ${ }^{[1]}$ Most patients in SA present with inguinal lymphadenopathy and bubo formation (the secondary phase, also termed the inguinal syndrome). In addition, proctitis and haemorrhagic procto-colitis (the anorectal syndrome) has been described in women and MSM who practise receptive anal intercourse. Currently, an outbreak of ano-rectal LGV is ongoing among MSM in Western Europe and, to a lesser extent, North America. ${ }^{\left[{ }^{[8]}\right.}$ The outbreak is associated with the newly described L2b variant of C. trachomatis. ${ }^{[9]}$ Many of these MSM are co-infected with HIV and it has been hypothesised that pathogenic C. trachomatis L2b strains may be passed between MSM on sex toys and other fomites. Ano-rectal LGV may also mimic inflammatory bowel disease, particularly Crohn's disease, on both clinical and histological grounds. ${ }^{[8]}$ Several reports exist of LGV in MSM who have been initially treated by gastroenterologists for inflammatory bowel disease. ${ }^{[10]}$ The diagnosis of LGV may be missed in such cases 
due to poor or non-existent sexual history taking by the attending physician.

In terms of LGV-associated lymph node histopathology, the presence of tiny necrotic foci with infiltrating neutrophils may be an early finding. These foci enlarge and coalesce to form the typical abscesses of LGV ${ }^{[2]}$ Epithelioid cells and Langhans-type giant cells line the abscess walls in later stages, and confluence of abscesses with sinus-formation can follow. ${ }^{[1,2]}$ Diagnosis of LGV is best undertaken using specific molecular DNA amplification assays and/or microimmunofluorescence serology. These diagnostic techniques are often only available in specialised reference laboratories. Most peripheral laboratories offer a CFT, but this assay lacks specificity. The CFT titres reported in this case are consistent with previous exposure to chlamydial infection and should not be regarded as indicative of a recent LGV infection. It should be appreciated that, as with syphilis, serology may be negative in the ulcerative stage and it is only a useful diagnostic tool in the context of patients presenting with the inguinal and ano-rectal syndromes.

\section{Conclusion}

Taking a detailed medical history is an important part of medical practice, including an adequate sexual history, particularly when a patient has a genital ulcer and inguinal lymphadenopathy. An adequate and relevant history can assist in directed special investigations and treatment. It is also important to remember that sexually transmitted diseases are best considered as a group, rather than individual entities, as they often occur together. The histological findings of the lymph node in the current case prompted the pathologist to request chlamydial serology. If relevant serologic tests had been included initially the diagnosis of syphilis could have been suspected and confirmed on Warthin-Starry stain. Histology of the foreskin ulcer would also have been beneficial in this case. This case highlights the value of a multidisciplinary approach in the management of such cases. Solving complicated cases such as these teaches us valuable lessons that can be used in managing patients in the future.

1. Rosai J. Chapter 21: Rosai and Ackerman's surgical pathology. In: Lymph Nodes Vol. 2. 10th ed. Elsevier, 2011:1785-1793

2. Ioachim HL, Medeiros LJ. Chapters 17, 19 and 20. In: Ioachim's Lymph Node Pathology. 4th ed. Philadelphia. Wolters Kluwer/Lippincott Williams \& Wilkins, 2009:110-114, 119-126.

3. Singh AE, Romanowski B. Syphilis: Review with emphasis on clinical, epidemiologic, and some biologic features. Clin Microbiol Rev 1999;12(2):187-209.

4. Fenton KA. A multilevel approach to understanding the resurgence and evolution of infectious syphilis in Western Europe. Euro Surveill 2004;9(12):3-4.

5. French P. Syphilis. BMJ 2007:334(7585):143-147. DOI:10.1136/bmj.39085.518148.BE

6. Heffelfinger ID, Swint EB, Berman SM, et al. Trends in primary and secondary syphilis among men who have sex with men in the United States. Am J Public Health 2007;97(6):1076-1083. DOI:10.2105/AJPH.2005.070417 Mabey D, Peeling RW. Lymphogranuloma venereum. Sex Transm Infect 2002;78(2):90-92. DOI:10.1136/sti.78.2.90

8. De Vrieze NH, de Vries HJ. Lymphogranuloma venereum among men who have sex with men. An epidemiological and clinical review. Expert Rev Anti Infect Ther 2014;12(6):697-704. DOI:10.1586/ 14787210.2014 .901169

9. Spaargaren J, Fennema HS, Morre SA, et al. New lymphogranuloma venereum Chlamydia trachomatis variant, Amsterdam. Emerg Infect Dis 2005;1 1 (7):1090-1092.

10. Soni $S$, Srirajaskanthan $R$, Lucas $S B$, et al. Lymphogranuloma venereum proctitis masquerading as inflammatory bowel disease in 12 homosexual men. Aliment Pharmacol Ther 2010;32(1):59-65. DOI:10.1111/j.1365-2036.2010.04313.x

Accepted 13 October 2014. 